ISSN 0103-9954

\title{
AUSÊNCIA DE ESTABILIZAÇÃO DA CURVA DE ACUMULAÇÃO DE ESPÉCIES EM FLORESTAS TROPICAIS
}

\author{
ABSENCE OF STABILIZATION POINT OF THE SPECIES ACCUMULATION \\ CURVE IN TROPICAL FORESTS
}

\author{
Ana Cristina Schilling ${ }^{1}$ João Luis Ferreira Batista ${ }^{2}$ Hilton Zarate do Couto ${ }^{3}$
}

\begin{abstract}
RESUMO
A definição do tamanho da amostra é um dos principais problemas em levantamentos fitossociológicos. A curva de acumulação de espécies é comumente utilizada para definir a suficiência amostral, mas este método apresenta algumas limitações, como a ausência de um ponto de estabilização que possa ser determinado objetivamente e a arbitrariedade da ordem de entrada das unidades amostrais na construção da curva. Uma solução para esse problema é o uso de procedimentos de aleatorização, como a permutação, para obter curvas médias de acumulação de espécies e seus intervalos de confiança empíricos. Entretanto, esse procedimento enfatiza o caráter assintótico da curva, que não apresenta ponto de inflexão, tornando-se impossível a determinação de um ponto objetivo para avaliar o tamanho ótimo da amostra.
\end{abstract}

Palavras-chave: curva de acumulação de espécies; fitossociologia; suficiência amostral.

\begin{abstract}
The definition of the sample size is a major problem in studies of phytosociology. The species accumulation curve is used to define the sampling sufficiency, but this method presents some limitations such as the absence of a stabilization point that can be objectively determined and the arbitrariness of the order of sampling units in the curve. A solution to this problem is the use of randomization procedures, e.g. permutation, for obtaining a mean species accumulation curve and empiric confidence intervals. However, the randomization process emphasizes the asymptotical character of the curve. Moreover, the inexistence of an inflection point in the curve makes it impossible to define objectively the point of optimum sample size.
\end{abstract}

Keywords: phytosociology; sampling sufficiency; species-accumulation curve.

\section{INTRODUÇÃO}

O número de espécies (também chamado riqueza de espécies) é o mais comum e mais simples descritor da diversidade biológica (GASTON, 1996). Porém, de acordo com Magurran (2004), a simplicidade dessa medida é ilusória, pois a quantificação objetiva da riqueza apresenta uma série de problemas, tais como o conceito de espécie a ser adotado, as dificuldades na discriminação das espécies e, particularmente, o processo de amostragem em si, pois geralmente é inviável inventariar toda a comunidade, ou mesmo definir seus limites.

A principal dificuldade nos estudos que visam estimar a riqueza de espécies de um local é determinar o quanto é necessário amostrar para se obter uma previsão acurada dessa variável, pois é importante saber se a amostra é grande o suficiente para permitir estimar com precisão a riqueza de espécies de uma

1. Engenheira Florestal, Dr ${ }^{\mathrm{a}}$., Professora Adjunta do Departamento de Ciências Exatas e Tecnológicas, Universidade Estadual de Santa Cruz, Rodovia Ilhéus-Itabuna, Km 16, CEP 45662-000, Ilhéus (BA). schiling@uesc.br

2. Engenheiro Florestal, Dr., Professor do Departamento de Ciências Florestais, Escola Superior de Agricultura Luiz de Queiróz, Universidade de São Paulo, Laboratório de Métodos Quantitativos, Av. Pádua Dias, 11, Caixa Postal 9, CEP 13418-900, Piracicaba (SP).parsival@usp.br

3. Engenheiro Agrônomo, Dr., Professor Titular do Departamento de Ciências Florestais, Escola Superior de Agricultura Luiz de Queiróz, Universidade de São Paulo, Laboratório de Métodos Quantitativos, Av. Pádua Dias, 11, Caixa Postal 9, CEP 13418-900, Piracicaba (SP). htzcouto@esalq.usp.br

Recebido para publicação em 13/03/2008 e aceito em 17/03/2011 
comunidade que, muitas vezes, não tem tamanho conhecido. Para isso, uma ferramenta ainda muito utilizada (SCHILLING e BATISTA, 2008) é a curva de amostragem, ou curva de acumulação de espécies (algumas vezes também chamada de curva do coletor), que mede a taxa em que novas espécies são adicionadas com o aumento do esforço amostral, dado pelo número de indivíduos observados ou por alguma medida substituta, como a área, o número de amostras ou o tempo (MAGURRAN, 2004). Nesse contexto, é importante ressaltar que há duas formas de curva espécie-área: as que relacionam o número de espécies com a área, para diferentes áreas (com limites bem definidos, como ilhas), descrevendo a riqueza de espécies de uma região, e as que descrevem a riqueza local, construídas com áreas crescentes (parcelas acumuladas) numa área relativamente homogênea. Somente essas últimas podem ser consideradas curvas de acumulação de espécies (CHAZDON et al., 1998; COLWELL e CODDINGTON, 1994; MAGURRAN, 2004).

No estudo de comunidades vegetais, a determinação da suficiência amostral (assumida como o ponto a partir do qual um aumento no esforço não aumenta significativamente a precisão da amostragem) está originalmente relacionada à ideia de área mínima, entendida como a "menor área que pode conter uma representação adequada da comunidade, tanto em relação ao número de espécies como à combinação característica dessas espécies" (BRAUN-BLANQUET, 1932; HOPKINS, 1957), que seria definida pela assíntota da curva espécie-área. Entretanto, Cain (1938) mostrou que a determinação visual de um patamar onde ocorre a estabilização é impossível, porque esse ponto depende das razões dos eixos usados para a construção da curva, propondo então a utilização de uma determinada inclinação da curva (em geral 10\%) para definir a área mínima. Porém Rice e Kelting (1955) demonstraram que esse valor muda constantemente, de acordo com o tamanho de amostra utilizado, tendo pouco valor como indicador de tamanho ótimo de amostra, ou da "suficiência amostral", já que, mesmo que a curva se torne horizontal, o ponto continua a mudar para a direita à medida que se aumenta o esforço amostral.

Alguns autores sustentam que a riqueza de espécies de árvores nos trópicos encontra um patamar entre 1 e 3 ha (BOOM, 1986; GENTRY, 1988; VAN DER MAAREL, 2005). Todavia, essa suposição, provavelmente, está baseada em trabalhos com florestas temperadas, onde as comunidades podem ser nitidamente delimitadas por características edáficas e climáticas bem definidas espacialmente. Nas florestas tropicais a definição de uma comunidade é bem menos nítida, e a grande quantidade de espécies raras faz com que a curva de acumulação de espécies tenda a crescer indefinidamente (CONDIT et al., 1996).

Cain (1956), em estudo realizado na Amazônia, em duas matas de terra firme na região de Belém, utilizando parcelas de 2 ha, não encontrou patamar para as curvas de acumulação de espécies desses locais. Oliveira (2000), numa avaliação de inventários florísticos realizados em matas de terra firme na Amazônia brasileira, concluiu que o método padrão de levantamento fitossociológico nessas áreas, utilizando parcelas de 1 ha, precisa ser mais bem avaliado, já que seus resultados de estrutura e composição não podem ser extrapolados para a floresta adjacente, mesmo em áreas muito próximas e em condições similares de topografia e solo. Condit et al. (1996), trabalhando com parcelas de 50 ha em três florestas tropicais distintas, em que foram medidos todos os indivíduos com mais de $1 \mathrm{~cm}$ de DAP, não observaram estabilização em nenhuma das curvas de acumulação de espécies.

A definição do tamanho ótimo da amostra através da curva de acumulação de espécies deve considerar a natureza assintótica dessa relação. Somente é possível determinar um ponto objetivo em uma curva assintótica se houver um ponto de inflexão, a partir do qual diminui a eficiência da amostragem. Para verificar a existência desse ponto na curva de acumulação de espécies podem-se utilizar os conceitos de incremento médio e marginal no número de espécies. $\mathrm{O}$ incremento marginal pode ser definido como o aumento na riqueza de espécies obtido com o aumento do tamanho da amostra, e o incremento médio é dado pela relação entre o número de espécies e determinado esforço amostral (PRODAN et al., 1997). A representação gráfica teórica dessas variáveis (Figura 1) permite observar que o ponto de máximo incremento médio ocorre na intersecção entre as duas curvas, posteriormente ao máximo incremento marginal, que definiria o ponto de inflexão da curva de acumulação de espécies. Ainda é possível verificar que, até seu ponto de máximo, o incremento médio está abaixo do marginal, e essa relação se inverte após esse ponto. Assim, cada unidade amostral acrescentada além do ponto de cruzamento das duas curvas dará um retorno abaixo da média obtida até o momento, indicando redução da eficiência da coleta. 


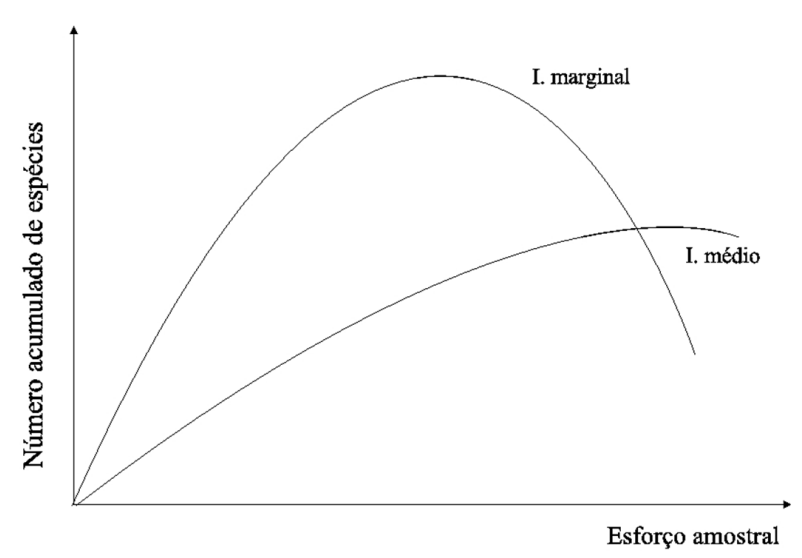

FIGURA 1: Relação entre incremento médio e incremento marginal na curva de acumulação de espécies.

FIGURE 1: Relationship between the average and marginal growth rates in the species accumulation curve.

Finalmente, outro aspecto limitante na determinação do tamanho ótimo da amostra através da curva de acumulação de espécies é a arbitrariedade da ordem de entrada das unidades amostrais, que já foi discutida por diversos autores (COLWELL e CODDINGTON, 1994; CHAZDON et al., 1998; MARTINS e SANTOS, 1999). Em princípio, cada possível ordenação das unidades pode gerar uma curva diferente e, assim, qualquer interpretação sobre a forma da curva gerada com uma dessas ordens é inválida. Do mesmo modo, se a ordem das unidades é arbitrária não pode ser utilizada como variável numa regressão, inviabilizando o uso de técnicas de modelagem para definir o ponto de estabilização da curva, como utilizado por alguns autores (GAMA et al., 2001, 2003; BENTESGAMA et al., 2002; BUDKE et al., 2004). Uma solução para o problema da arbitrariedade da ordem das unidades na construção da curva é a utilização de uma curva suavizada (CHAZDON et al., 1998; MAGURRAN, 2004) obtida por procedimentos de aleatorização, como a permutação (EFRON e TIBSHIRANI, 1993), em que um grande número de curvas é obtido a partir de vários ordenamentos distintos, permitindo obter a curva média e o intervalo de confiança empírico para essa curva.

A escolha do tipo de curva a ser utilizada, baseada em indivíduos ou amostras, é função dos dados disponíveis. Se a coleta for baseada em indivíduos não há alternativa, mas se for baseada em amostras pode-se construir os dois tipos de curva. Segundo Gotelli e Colwell (2001), em geral é preferível construir curvas baseadas em amostras, para que sejam considerados os níveis naturais de heterogeneidade da área, principalmente quando as espécies ocorrem muito agrupadas. Por outro lado, quando o objetivo é comparar a riqueza de espécies entre locais, Condit et al. (1996) e Gotelli e Colwell (2001) sustentam que curvas de acumulação de espécies, baseadas em indivíduos, são mais adequadas, pois os conjuntos de dados podem diferir, sistematicamente, no número médio de indivíduos por amostra, e então as diferenças em riqueza poderiam ter sido ocasionadas pelas diferenças em densidade de cada local.

Mesmo com todas as limitações e recomendações contrárias, o uso da curva de acumulação de espécies (ou curva do coletor) continua sendo uma prática bastante comum para determinar a suficiência amostral em estudos de ecologia vegetal, particularmente no Brasil (ASSUNÇÃO e FELFILI, 2004; COSTA, 2004; DORNELES e WAECHTER, 2004; SILVA e SCARIOT, 2004). O conceito de suficiência amostral está relacionado com a qualidade da representação da composição florística, obtida com a amostragem e, estatisticamente, é interpretada como a precisão da informação gerada pela amostra. Porém, a definição estatística de tamanho ótimo de amostra combina a precisão desejada com o custo de obtenção da informação. Assim, o tamanho ótimo da amostra será aquele que maximize a precisão, mantendo o custo constante ou, de forma equivalente, minimize o custo mantendo a precisão constante (SHIVER e BORDERS, 1996). Considerando a composição florística das florestas tropicais, com predominância de espécies raras, não há ponto de estabilização da curva em termos do número de espécies que são observadas à medida que se aumenta o tamanho da amostra (CONDIT et al., 1996) e, se um dos fatores não for fixado (custo ou precisão), o tamanho da amostra tenderá ao infinito.

Assim, o objetivo deste trabalho é demonstrar empiricamente a ausência de patamar na curva de acumulação de espécies arbóreas em florestas tropicais e, dessa forma, a inadequação do uso da curva de acumulação de espécies para determinar a suficiência amostral.

\section{MATERIAL E MÉTODO}

\section{Áreas de estudo}

Os dados utilizados nesse trabalho são provenientes de três Unidades de Conservação do 
Estado de São Paulo, que representam diferentes formações florestais da região tropical: Floresta Ombrófila Densa Submontana (Parque Estadual de Carlos Botelho), Floresta Estacional Semidecidual (Estação Ecológica dos Caetetus) e Cerrado lato sensu ou Savana florestada (Estação Ecológica de Assis), onde foram instaladas as grades de amostragem do Projeto "Métodos de Inventário da Biodiversidade de Espécies Arbóreas", do Programa Biota da FAPESP.

A Estação Ecológica de Assis situa-se numa área de domínio do cerrado, com área de vegetação natural (cerrado e cerradão) de 1.600 ha, localizada entre as coordenadas $22^{\circ} 33^{\prime}$ e $22^{\circ} 36^{\prime} \mathrm{S}$ e $50^{\circ} 22^{\prime}$ e $50^{\circ} 23^{\prime} \mathrm{W}$, e com altitudes variando de 520 a $590 \mathrm{~m}$. A área sofreu pastoreio intensivo e exploração de madeira até o início da década de 70 , quando passou a ser protegida. Foi incorporada à Estação Experimental de Assis em 1982 e em 1992 transformada em Estação Ecológica, estando assegurada por lei a sua preservação definitiva (DURIGAN, 1994).

A Estação Ecológica de Caetetus é considerada uma das maiores áreas com vegetação natural em bom estado de conservação em todo o Centro-Oeste do Estado de São Paulo. A estação tem área de 2.178 ha, com topografia ondulada e altitude variando de 500 a $680 \mathrm{~m}$, entre as coordenadas $22^{\circ} 22^{\prime}$ e $22^{\circ} 27^{\prime}$ S e $49^{\circ} 40^{\prime}$ e $49^{\circ} 43^{\prime} \mathrm{W}$. A vegetação é de Floresta Estacional Semidecidual, com altura do dossel entre 25 e $30 \mathrm{~m}$ e alta densidade de plantas herbáceoarbustivas no sub-bosque. (ROCHA, 2003).

O Parque Estadual de Carlos Botelho, localizado na região sul do estado de São Paulo, é um remanescente bem preservado de Floresta Ombrófila Densa Submontana, dentro dos domínios da Mata Atlântica, com área total de 37.797 ha. O relevo é acidentado, a altitude varia entre 30 e 1003 $\mathrm{m}$ e localiza-se entre as coordenadas $24^{\circ} 00^{\prime}$ e $24^{\circ} 15^{\prime}$, S e $47^{\circ} 45^{\prime}$ e $48^{\circ} 10^{\prime} \mathrm{W}$ (DIAS, 2005).

\section{Amostragem}

As grades de amostragem, instaladas nas três áreas, consistem de uma malha de $8 \times 8$ pontos distribuídos sistematicamente a cada 100 metros (Figura 2). Os 64 pontos de cada grade foram georreferenciados e monumentados no campo.

Em cada grade há 64 parcelas retangulares de $90 \times 10 \mathrm{~m}\left(900 \mathrm{~m}^{2}\right)$ e em cada uma delas foram contados e identificados todos os indivíduos com diâmetro (DAP) maior ou igual a $5 \mathrm{~cm}$. Cada linha de amostragem é formada por 8 parcelas que, por sua vez, são divididas em 9 subparcelas de $10 \times 10 \mathrm{~m}$ $\left(100 \mathrm{~m}^{2}\right)$, com um intervalo de $10 \mathrm{~m}$ não amostrado entre as parcelas nas linhas. Dessa forma, cada grade tem uma área de abrangência de 64 ha, porém com uma área efetivamente amostrada de 5,76 ha.

\section{Análise}

\section{Curvas de acumulação de espécies}

Os levantamentos florísticos referentes às grades de amostragem, de cada uma das áreas, foram usados para produzir matrizes de presença/ausência de espécies, com as quais foram obtidas as curvas usuais de acumulação de espécies, as curvas médias aleatorizadas e os intervalos de confiança empíricos em nível de parcela. As curvas de acumulação de espécies baseadas em indivíduos (usuais, médias e intervalos empíricos) foram obtidas através das matrizes de indivíduos por espécie, conforme Batista e Schilling (2006).

As curvas usuais, resultantes da acumulação das espécies na ordem de coleta dos dados (parcelas ou árvores) foram construídas observando-se a ordem das parcelas nas linhas das grades, para preservar a estrutura espacial dos dados. As curvas médias, independentes da ordem de entrada dos dados, e os intervalos de confiança empíricos, utilizando-se os quantis 2,5 e $97,5 \%$, foram obtidos através de reamostragem por permutação (EFRON e TIBSHIRANI, 1993). Para cada grade foram geradas 1.000 simulações, com ordem aleatória, em nível de unidade amostral (parcelas e árvores).

\section{Curvas de incremento médio e marginal}

A partir do vetor com os valores médios de riqueza de espécies, obtidos pelo procedimento de aleatorização descrito acima, foram construídos os gráficos de incremento médio e marginal no número de espécies. O incremento médio é obtido pela divisão do número de espécies pelo número de unidades amostrais observadas, e o incremento marginal expressa o aumento no número de espécies à medida que as unidades amostrais são acumuladas (PRODAN et al., 1997).

\section{RESULTADOS E DISCUSSÃO}

Os biomas estudados (savana florestada, floresta estacional semidecidual e floresta ombrófila densa) representam um gradiente ambiental com influência crescente do deficit hídrico e na Tabela 1 pode-se observar um aumento na riqueza de espécies 


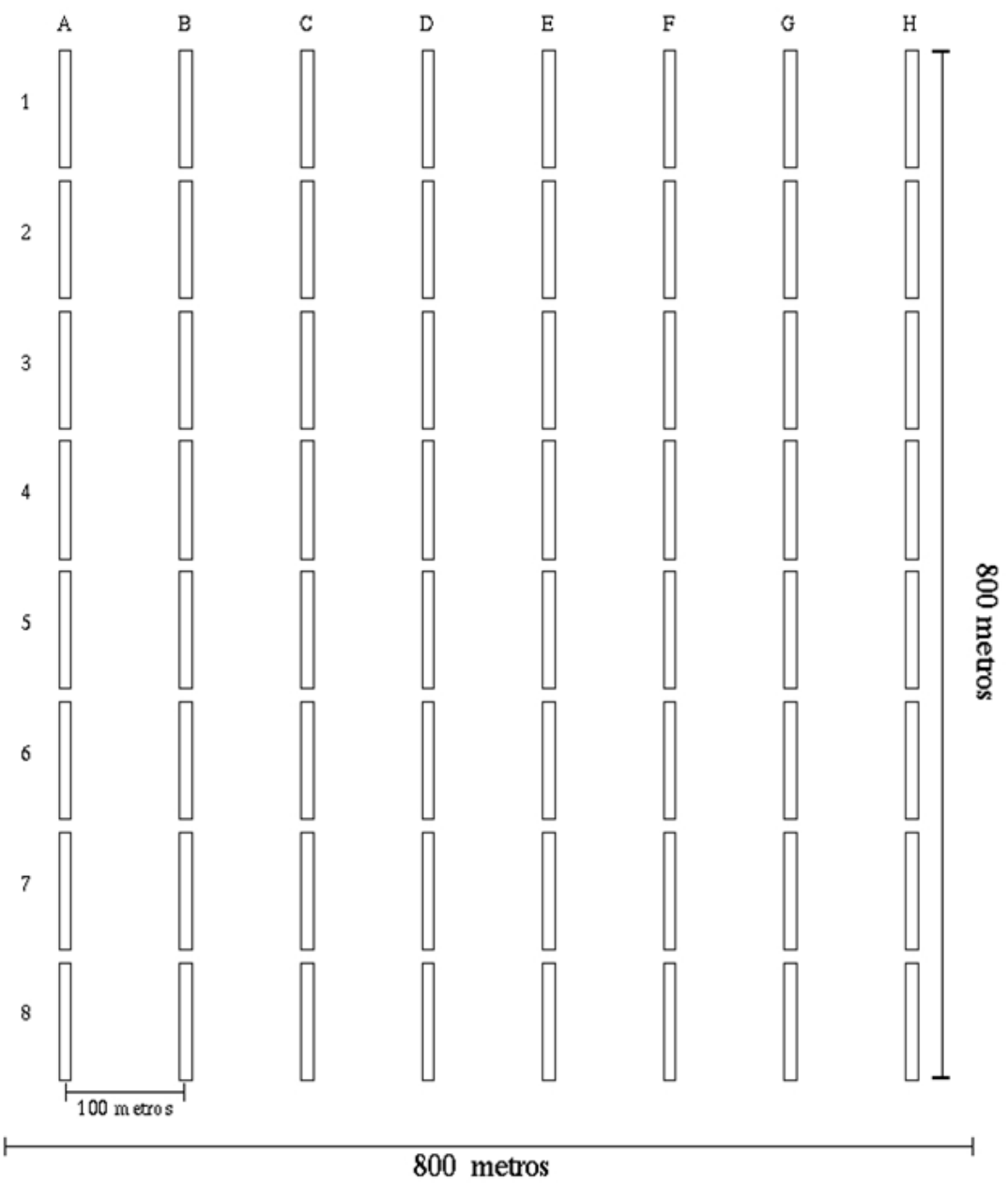

FIGURA 2: Esquema das grades de amostragem utilizadas no estudo, representando as parcelas retangulares de $900 \mathrm{~m}^{2}$.

FIGURE 2: Sampling grids used in the study area showing the position of the rectangular plots of $900 \mathrm{~m}^{2}$.

ao longo desse gradiente. Além disso, a Estação Ecológica de Assis (savana florestada) apresenta uma grande densidade de árvores e menor riqueza de espécies quando comparada às outras áreas.

Na Figura 3 são apresentadas as curvas de acumulação de espécies por parcela, para as três áreas do estudo. Em nenhuma das áreas foi possível observar a ocorrência de patamar nas curvas, particularmente nas construídas por aleatorização, mesmo com um esforço amostral considerado grande para esse tipo de estudo (64 parcelas de $900 \mathrm{~m}^{2}$ e área efetiva de 5,76 ha), que é feito predominantemente em parcelas de 1 ha (OLIVEIRA, 2000; MARTINS, 1989). As curvas de acumulação de espécies por indivíduo são apresentadas na Figura 4, também para as três áreas. Da mesma forma que nas curvas de acumulação por parcelas, nenhum patamar é verificado.
TABELA 1: Número de árvores e espécies amostradas nas grades amostrais das três formações florestais avaliadas.

TABLE 1: Number of sampled trees and species in sampling grids of three forest formations.

\begin{tabular}{lll}
\hline Unidade de Conservação & $\begin{array}{l}\text { Número de } \\
\text { Árvores }\end{array}$ & $\begin{array}{l}\text { Número de } \\
\text { Espécies }\end{array}$ \\
\hline
\end{tabular}

Estação Ecológica de Assis (Cerradão)

$10.929 \quad 104$

Estação Ecológica de Caetetus

(Floresta Estacional

7.520

Semidecidual)

Parque Estadual de Carlos

Botelho $\quad 9.544 \quad 253$

(Floresta Ombrófila Densa) 


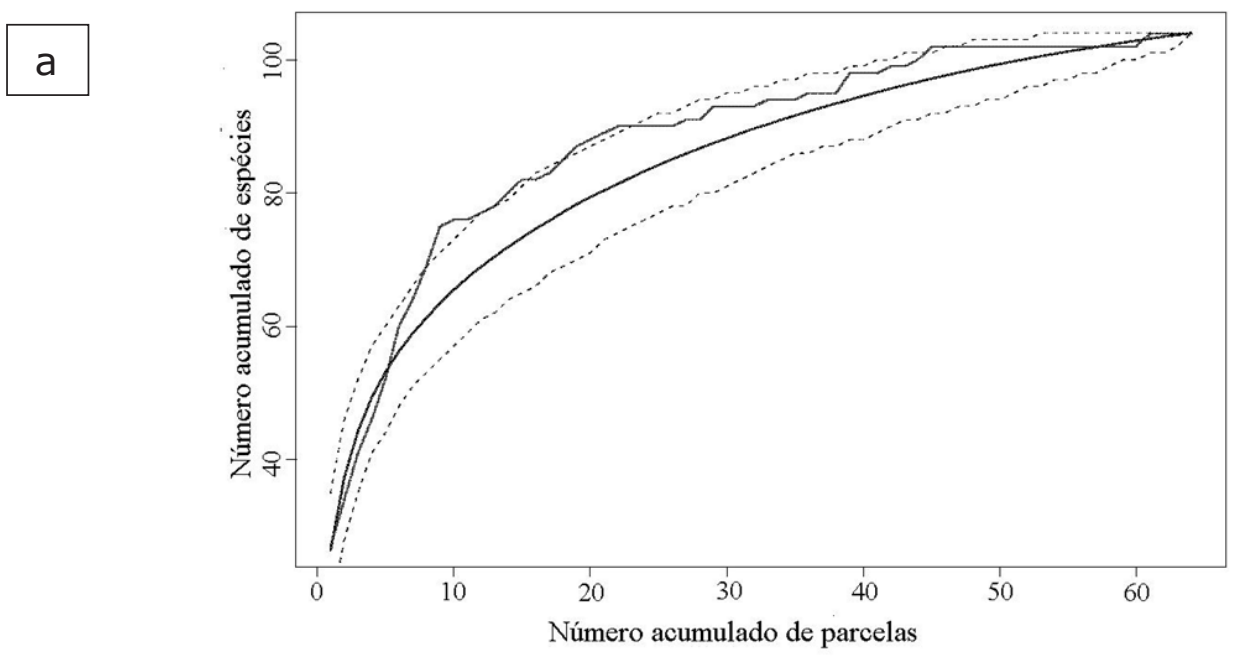

$\mathrm{b}$

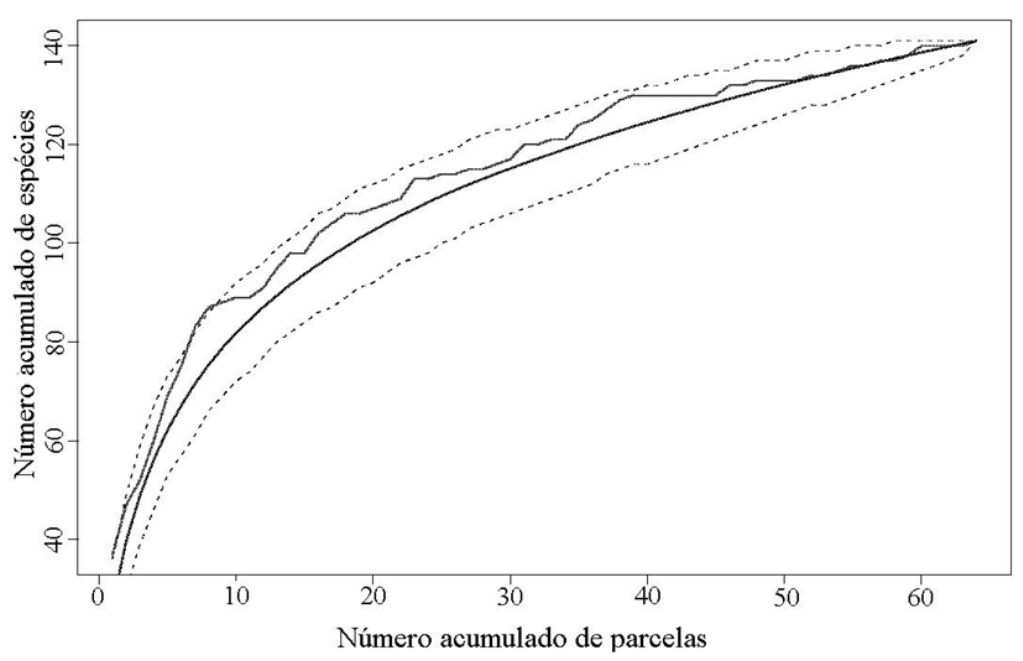

C

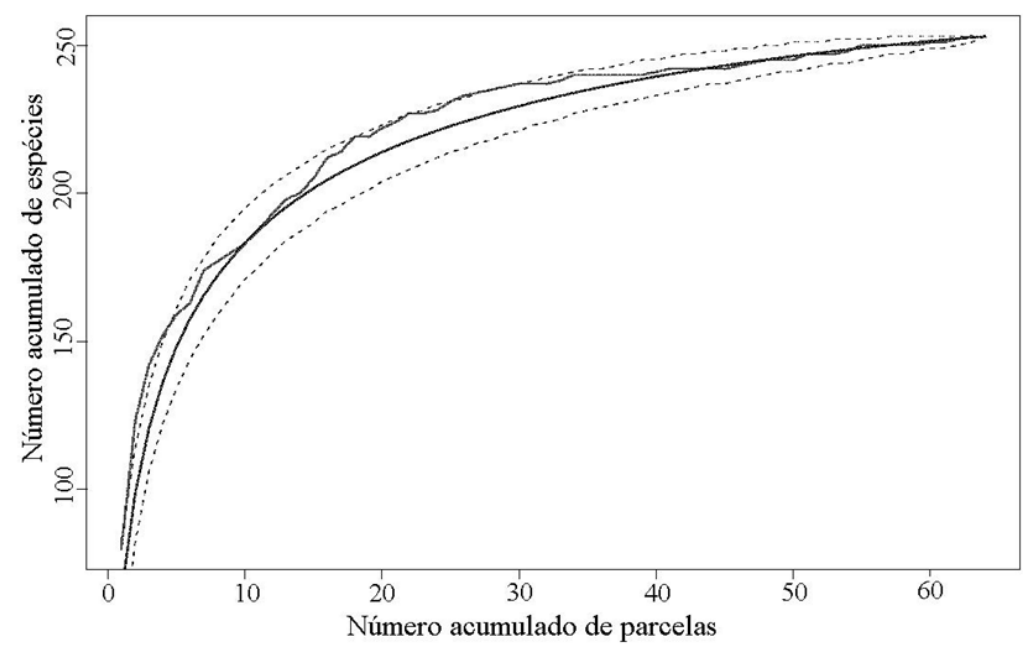

FIGURA 3: Curvas médias de acumulação de espécies (linhas contínuas suavizadas), intervalos de confiança de 95\% (linhas tracejadas) e as curvas usuais, utilizando a ordem das parcelas (linhas irregulares). (a) E. E. de Assis, (b) E. E. de Caetetus e (c) P. E. de Carlos Botelho.

FIGURE 3: Average species accumulation curves (continuous, smoothed lines), 95\% confidence intervals (hatched lines) and usual curves, using the observation order of plots (irregular lines). (a) E. E. de Assis, (b) E. E. de Caetetus e (c) P. E. de Carlos Botelho. 

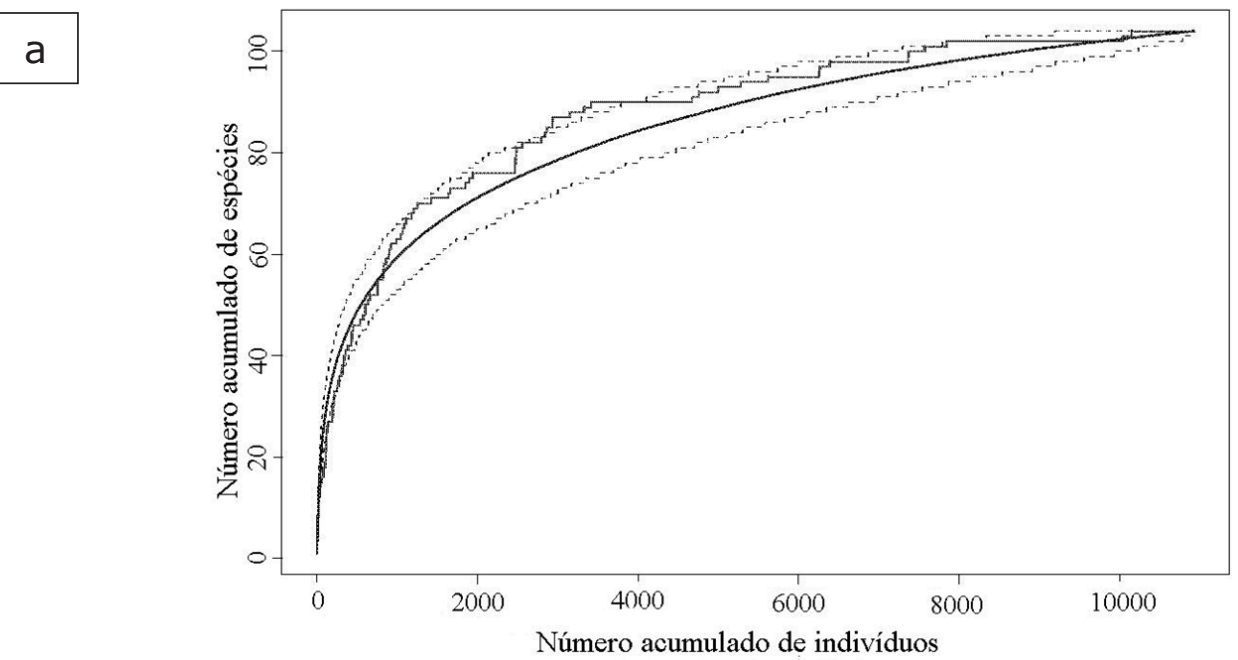

b

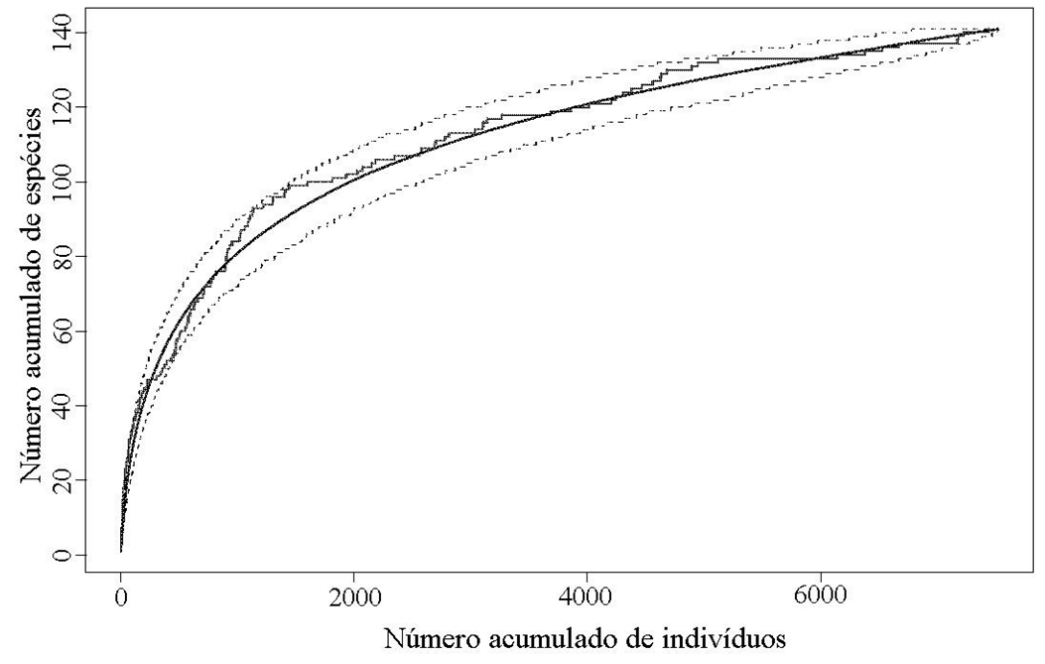

C

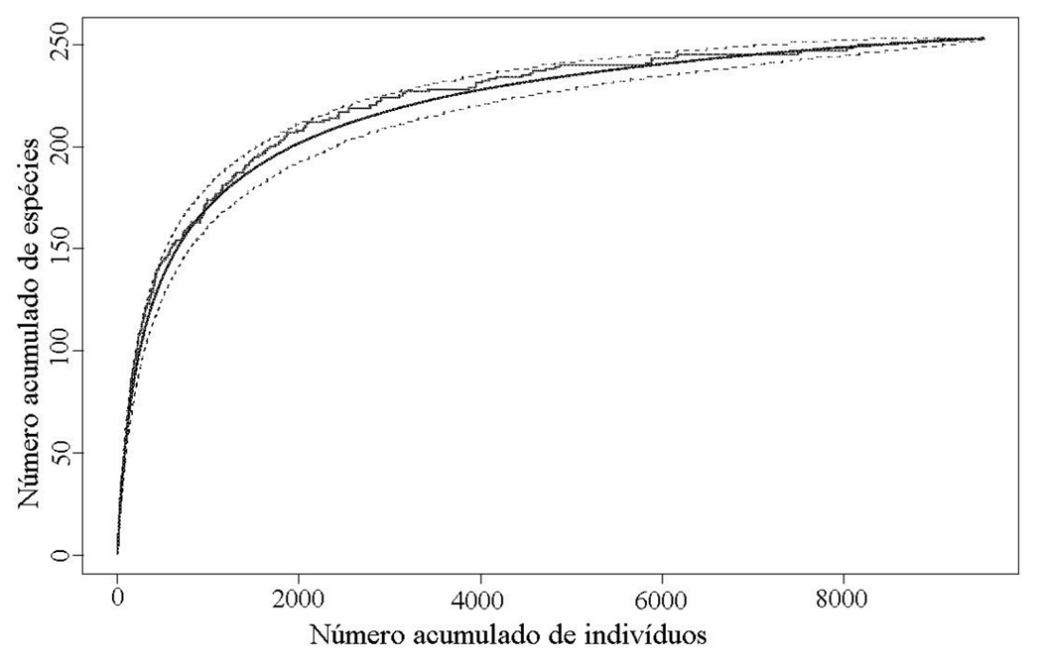

FIGURA 4: Curvas médias de acumulação de espécies (linhas contínuas suavizadas), intervalos de confiança de $95 \%$ (linhas tracejadas) e as curvas usuais, utilizando a ordem de observação dos indivíduos (linhas irregulares). (a) E. E. de Assis, (b) E. E. de Caetetus e (c) P. E. de Carlos Botelho.

FIGURE 4: Average species accumulation curves (continuous, smoothed lines), 95\% confidence intervals (hatched lines) and usual curves, using the observation order of individuals (irregular lines). (a) E. E. de Assis, (b) E. E. de Caetetus e (c) P. E. de Carlos Botelho. 
Pode-se observar que, as curvas usuais de acumulação de espécies obtidas na ordem de observação dos dados (seja acumulação de parcelas ou de indivíduos), podem algumas vezes apresentar indícios de estabilização (Figuras 3 e 4, linhas irregulares). Porém, como essa ordem é arbitrária, a forma dessa curva não representa $o$ comportamento da taxa de acumulação de espécies nessas comunidades, pois qualquer outra ordem dos dados resultaria numa curva diferente e, consequentemente, em um ponto de estabilização também diferente.

Como é inadequado determinar o tamanho ideal da amostra baseado em uma informação completamente dependente de um fator arbitrário como o caminho percorrido na floresta para a coleta, a curva usual mostra-se ineficiente. Da mesma forma, o uso de técnicas de ajuste da curva por regressão linear ou não linear, para tentar estimar o ponto de estabilização da curva, também não é válido, pois está baseado numa ordem arbitrária das unidades amostrais, em que cada ordenação dos dados resultaria num modelo de regressão diferente.

$O$ uso da curva média obtida por permutação (EFRON e TIBSHIRANI, 1993) pressupõe a completa aleatoriedade na ocorrência das espécies, o que em geral não é observado nas formações florestais dos trópicos. Nessas formações as espécies geralmente ocorrem agrupadas, fazendo com que a curva média, gerada por aleatorização, tenha uma inclinação menor que a curva observada. Assim, quanto maior a discrepância entre a curva usual e a curva média, maior a evidência de um efeito estrutural da comunidade. Nas Figuras 3 e 4 pode-se observar que todas as curvas médias tiveram menor curvatura que as respectivas curvas usuais, e que estas extrapolaram (por acréscimo ou decréscimo) os intervalos de confiança empíricos em diversos pontos, evidenciando algum grau de agrupamento na ocorrência das espécies. Além disso, as curvas médias obtidas por aleatorização têm seu caráter assintótico enfatizado, tornandose impossível observar qualquer tendência de estabilização (Figuras 3 e 4, linhas suavizadas), pois somente é possível definir um ponto objetivo sobre uma curva assintótica se essa curva apresentar um ponto de inflexão.

Na Figura 5 são apresentados os gráficos com o incremento médio e marginal de inclusão de novas espécies para as três áreas em estudo. $\mathrm{Na}$ primeira coluna são apresentados os gráficos construídos com o número acumulado de parcelas como medida do esforço amostral, e na segunda coluna, a medida utilizada foi o número acumulado de indivíduos.

Em nenhum dos casos ocorre o cruzamento das curvas de incrementos médio e marginal. Nas curvas de incremento construídas com o esforço amostral, medido em número acumulado de indivíduos, observa-se uma sobreposição inicial das duas curvas, pois para um único indivíduo amostrado, naturalmente o incremento médio será igual ao marginal.

Conforme Prodan et al. (1997) o ponto de cruzamento das curvas de incremento médio e marginal define o ponto de máximo incremento médio, a partir do qual a curva se tornaria assintótica, e isso ocorre num ponto posterior ao máximo incremento marginal. As relações entre essas curvas podem ser derivadas matematicamente, e é possível demonstrar que o máximo incremento marginal corresponde ao ponto de inflexão na curva de acumulação de espécies. Como as curvas dos incrementos não se cruzam em nenhum ponto, pode-se dizer que a curva de acumulação de espécies é assintótica, porém, sem apresentar um ponto de inflexão. Deve-se salientar ainda que a forma assintótica também se aplica à banda de confiança, delimitada por duas linhas paralelas à curva média de acumulação de espécies, de modo que a incerteza sobre a acumulação de espécies se manifesta também sobre o valor verdadeiro da assíntota, e sobre a velocidade com que se aproxima da assíntota. Assim, não é possível definir um ponto objetivo na curva de acumulação de espécies a partir do qual ocorre a estabilização, e que seria o tamanho ótimo da amostra.

\section{CONCLUSÕES}

Mesmo quando o patamar da curva usual de acumulação de espécies (construída na ordem de coleta dos dados) aparentemente é obtido, a curva média obtida pela aleatorização dos mesmos dados não apresenta ponto de estabilização, já que o caráter assintótico da curva é enfatizado por esse procedimento. As curvas usuais de acumulação de espécies apresentaram maior curvatura que suas respectivas curvas médias, o que evidencia a falta de aleatoriedade na ocorrência das espécies.

A ausência de cruzamento entre as curvas de incrementos médio e marginal, no número de espécies, demonstra que não há ponto de inflexão na curva de acumulação de espécies, o que inviabiliza 

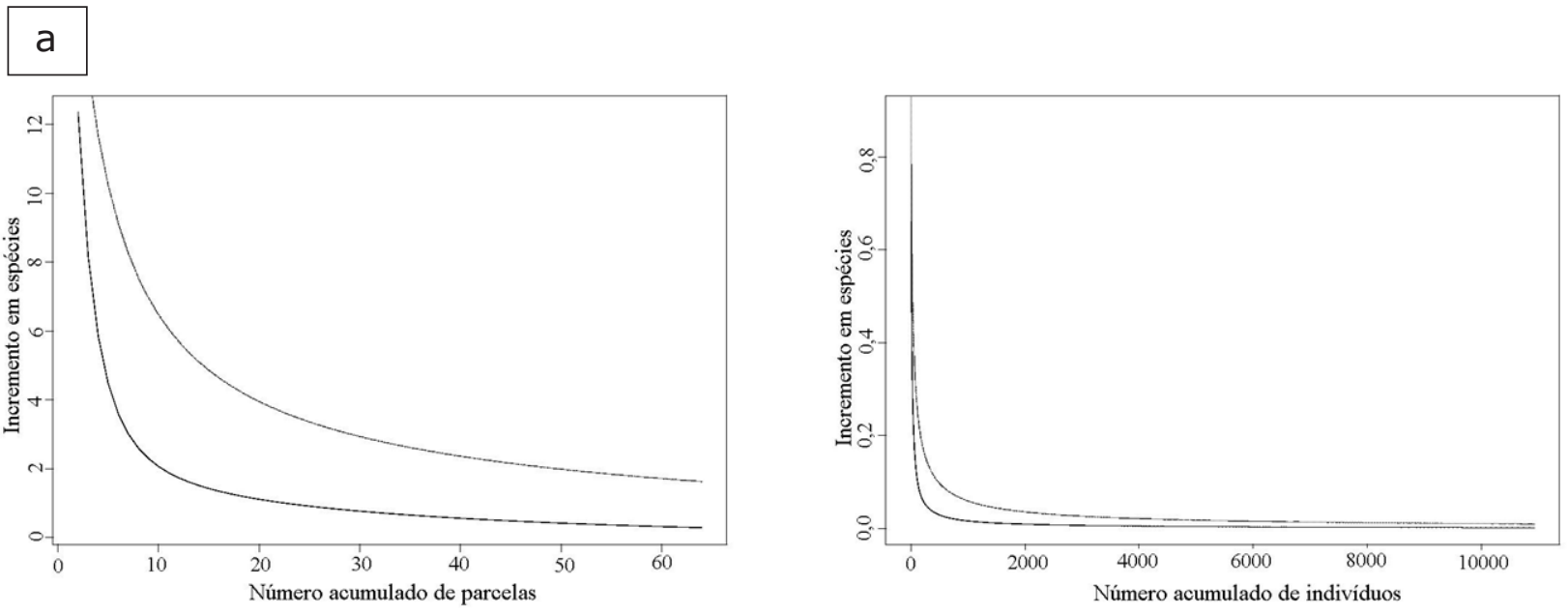

\section{b}
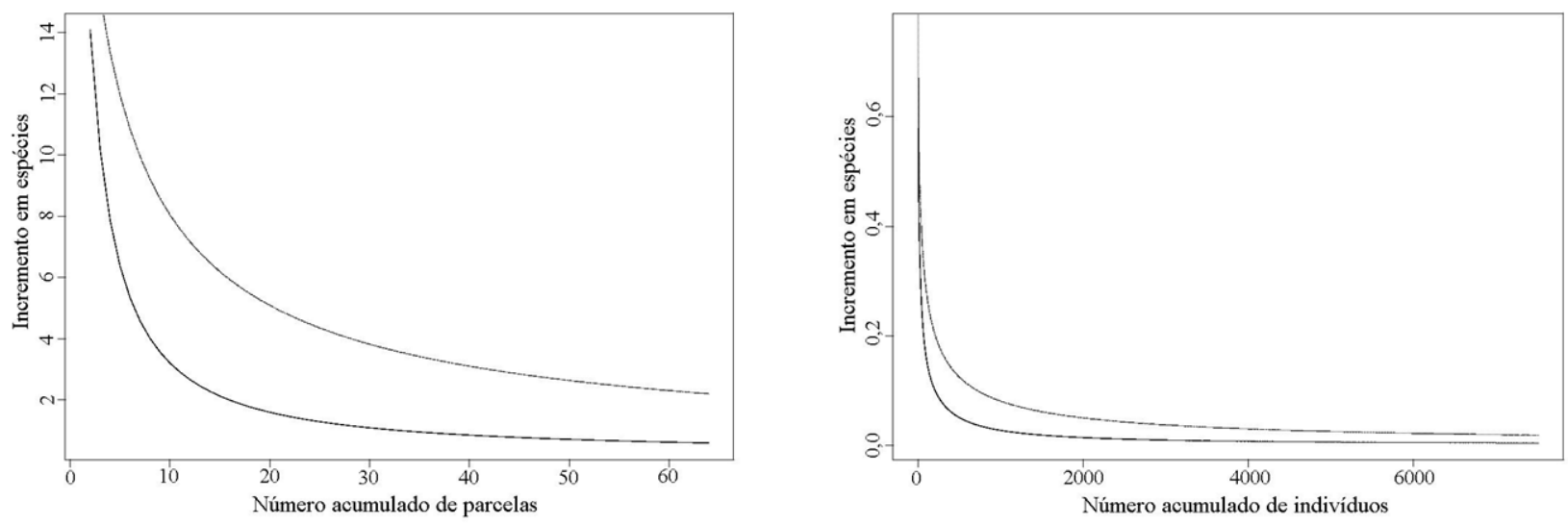

\section{c}
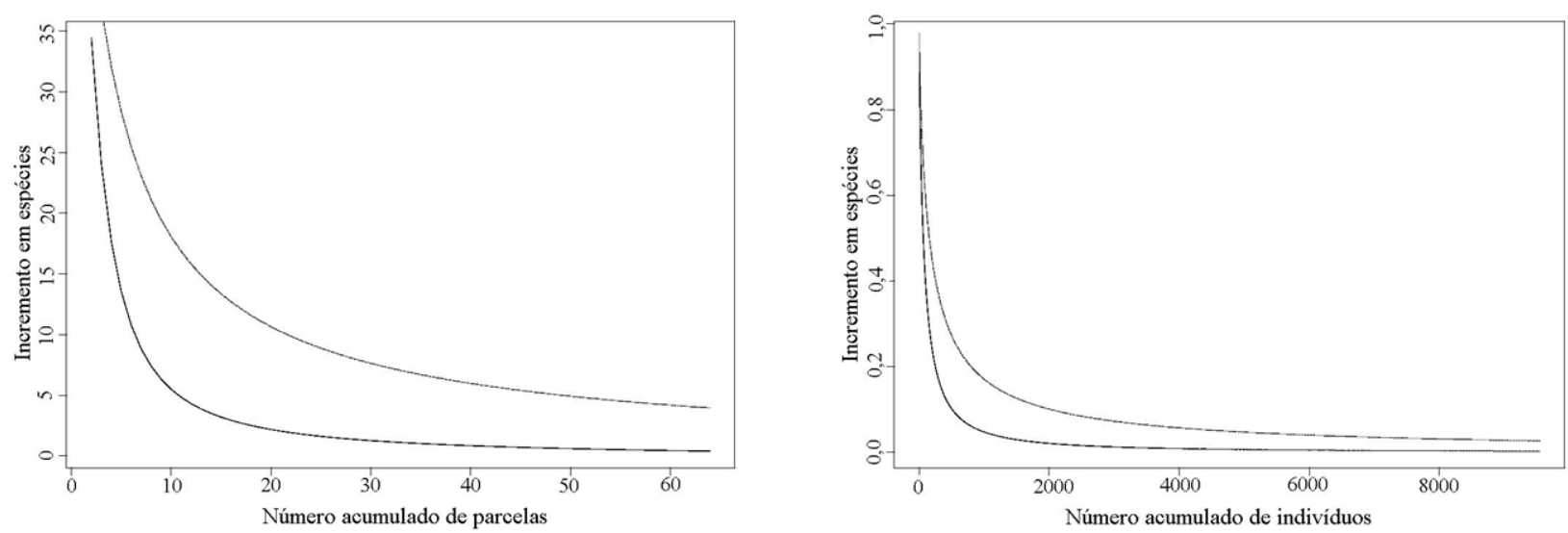

FIGURA 5: Curvas de incremento médio (linha inferior) e incremento marginal (linha superior) na inclusão de novas espécies, para (a) E.E. de Assis, (b) E.E. de Caetetus, e (c) P. E. de Carlos Botelho.

FIGURE 5: Average growth (lower lines) and marginal growth (upper lines) curves in the species accumulation curve for (a) E.E. de Assis, (b) E.E. de Caetetus, and (c) P. E. de Carlos Botelho. 
a determinação de um ponto objetivo sobre a curva assintótica.

A agregação de espécies é uma característica intrínseca das florestas tropicais, o que contraria a premissa de completa aleatoriedade necessária para a utilização de uma curva média de acumulação de espécies para determinar a suficiência amostral.

\section{AGRADECIMENTOS}

Os autores agradecem à Fundação de Amparo à Pesquisa do Estado de São Paulo (FAPESP), pelo financiamento do projeto "Métodos de Inventário da Biodiversidade", do programa Biota. À Adriana Martini, Fernando Roberto Martins, Paulo Inácio Prado e Sergius Gandolfi pelas valiosas sugestões na elaboração do trabalho. Finalmente, a autora agradece a Fundação de Amparo à Pesquisa do Estado da Bahia (FAPESB) pela concessão da bolsa de doutorado.

\section{REFERÊNCIAS BIBLIOGRÁFICAS}

ASSUNÇÃO, S. L.; FELFILI, J. M. Fitossociologia de um fragmento de cerrado senso stricto na APA do Paranoá, DF, Brasil. Acta Botanica Brasilica, São Paulo, v. 18, p. 903-909, 2004.

BATISTA, J. L. F.; SCHILLING, A. C. S. Um algoritmo matricial para construção da curva de acumulação de espécies. Disponível em (http://lmq. esalq.usp.br/METRVM/) > Acesso em: 25 de julho de 2006.

BENTES-GAMA, M. M. et al.. Estrutura e valoração de uma floresta de várzea alta na Amazônia. Cerne, Lavras, v. 8, p. 88-102, 2002.

BOOM, B. M. A forest inventory in Amazonian Bolivia. Biotropica, Lawrence, v. 18, p. 287-294. 1986.

BRAUN-BLANQUET, J. Plant sociology. New York: McGraw Hill, 1932. 439 p.

BUDKE, J. C. et al. Florística e fitossociologia do componente arbóreo de uma floresta ribeirinha, arroio Passo das Tropas, Santa Maria, RS, Brasil. Acta Botanica Brasilica, São Paulo, v. 18, p. 581589, 2004.

CAIN, S. A. The species-area curve. The American Midland Naturalist, Notre Dame, v. 19, p. 573581, 1938.

CAIN, S. A. Application of some phytosociological techniques to brazilian rain forest. American Journal of Botany, St. Louis, v. 43, p. 911-941, 1956.
CHAZDON, R. L. et al. Statistical methods for estimating species richness of woody regeneration in primary and secondary rain forests of northeastern Costa Rica. In: DALLMEIER, F. B.; COMISKEY, J. A. (Ed.) Forest biodiversity research, monitoring and modeling: conceptual background and old world case studies. Paris: Parthenon Publishing, 1998. Chap.16. p.285-309.

COLWELL, R. K.; CODDINGTON, J.A. Estimating terrestrial biodiversity through extrapolation. Philosophical Transactions of the Royal Society of London, London, v. B345, p. 101-118, 1994.

CONDIT, R. et al. Species-area and speciesindividual relationships for tropical trees: a comparison of three 50-ha plots. Journal of Ecology, London, v. 84, p. 549-562, 1996.

COSTA, F. R. C. Structure and composition of the ground-herb community in a terra-firme Central Amazonian forest. Acta Amazonica, Manaus, v. 34, p. 53-59, 2004.

DIAS, A. C. Composição florística, fitossociologia, diversidade de espécies arbóreas e comparação de métodos de amostragem na floresta ombrófila densa do Parque Estadual de Carlos Botelho/ SPBrasil. 2005. 202 f. Tese (Doutorado em Recursos Florestais)-Escola Superior de Agricultura "Luiz de Queiroz", Universidade de São Paulo, Piracicaba, 2005.

DORNELES, L. P.; WAECHTER, J. L. Fitossociologia do componente arbóreo na floresta turfosa do Parque Nacional da Lagoa do Peixe, Rio Grande do Sul, Brasil. Acta Botanica Brasilica, São Paulo, v. 18, p. 815-824, 2004.

DURIGAN, G. Florística, fitossociologia e produção de folhedo em matas ciliares da região oeste do estado de São Paulo. 1994. 161 f. Tese (Doutorado em Biologia Vegetal)-Instituto de Biologia, Universidade Estadual de Campinas, Campinas, 1994.

EFRON, B.; TIBSHIRANI, R. J. An introduction to the bootstrap. New York: Chapman \& Hall, 1993. $436 \mathrm{p}$.

GAMA, J. R. V. et al. Tamanho de parcela e suficiência amostral para estudo da regeneração natural de floresta de várzea na Amazônia. Cerne, Lavras, v. 7, p. 1-11, 2001.

GAMA, J. R. V. et al. Estrutura e potencial futuro de utilização da regeneração natural de floresta de várzea alta no município de Afuá, Estado do Pará. Ciência Florestal, Santa Maria, v. 13, p. 71-82, 2003.

GASTON, K. J. Species richness: measure and 
measurement. In: GASTON, K. J.(Ed.) Biodiversity, a biology of numbers and difference. Oxford: Blackwell Science, 1996. Chap.4. p.77-113.

GENTRY, A. H. Changes in plant community diversity and floristic composition on environmental and geographical gradients. Annals of the Missouri Botanical Garden, St. Louis, v. 75, p. 1-34, 1988. GOTELLI, N. J.; COLWELL, R. K. Quantifying biodiversity: procedures and pitfalls in the measurement and comparison of species richness. Ecology Letters, Edinburgh, v. 4, p. 379-391, 2001. HOPKINS, B. The concept of minimal area. Journal of Ecology, London, v. 45, p. 441-449, 1957.

MAGURRAN, A. E. Measuring biological diversity. Oxford: Blackwell Publishing, 2004. 256 $\mathrm{p}$.

MARTINS, F. R. Fitossociologia de florestas do Brasil -- um histórico bibliográfico. Pesquisas Série Botânica, São Leopoldo, v. 40, p. 103-164, 1989.

MARTINS, F. R.; SANTOS, F. A. M. Técnicas usuais de estimativa da biodiversidade. Holos, Campinas, v. 1, p. 236-267, 1999.

OLIVEIRA, A. A. Inventários quantitativos de árvores em matas de terra firme: histórico com enfoque na Amazônia brasileira. Acta Amazonica, Manaus, v. 30, p. 543-567, 2000.
PRODAN, M. et al. Mensura forestal. San José: Deustche Gesellschaft für Technische Zusammenarbeit (GTZ): Instituto Interamericano de Cooperación para la Agricultura (IICA), 1997. $586 \mathrm{p}$.

RICE, E. L.; KELTING, R. W. The species-area curve. Ecology, Washington, v. 36, p. 7-11, 1955.

ROCHA, F. T. Levantamento florestal na Estação Ecológica dos Caetetus como subsídio para laudos de desapropriação ambiental. 2003. 172 f. Dissertação (Mestrado em Recursos Florestais)Escola Superior de Agricultura "Luiz de Queiroz", Universidade de São Paulo, Piracicaba, 2003.

SCHILLING, A. C.; BATISTA, J. L. F Curva de acumulação e suficiência amostral em florestas tropicais. Revista Brasileira de Botânica, São Paulo, v. 31, p. 179-187, 2008.

SHIVER, B. D.; BORDERS, B. E. Sampling techniques for forest resource inventory. New York: John Wiley, 1996. 356 p.

SILVA, L. A.; SCARIOT, A. Composição e estrutura da comunidade arbórea de uma floresta estacional decidual sobre o afloramento calcário no Brasil Central. Revista Árvore, Viçosa, v. 28, p. 69-75, 2004.

VAN DER MAAREL, E. Vegetation ecology. Malden: Blackwell Publishing, 2005. 395 p. 